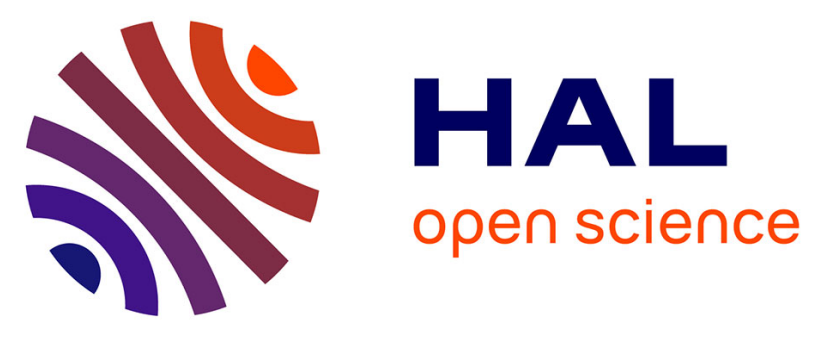

\title{
Subtyping of Primary Aldosteronism in the AVIS-2 Study: Assessment of Selectivity and Lateralization
}

Giacomo Rossitto 1 2, Laurence Amar 3, Michel Azizi 3, Anna Riester 4, Martin Reincke 4, Christoph Degenhart 4, Jiri Widimsky 5, Mitsuhide Naruse 6, Jaap Deinum 7, Leo Schultzekool 7, et al.

\section{To cite this version:}

Giacomo Rossitto 1 2, Laurence Amar 3, Michel Azizi 3, Anna Riester 4, Martin Reincke 4, et al.. Subtyping of Primary Aldosteronism in the AVIS-2 Study: Assessment of Selectivity and Lateralization. JOURNAL OF CLINICAL ENDOCRINOLOGY \& METABOLISM, 2020. hal-03108429

\section{HAL Id: hal-03108429 \\ https://hal.science/hal-03108429}

Submitted on 25 Jan 2021

HAL is a multi-disciplinary open access archive for the deposit and dissemination of scientific research documents, whether they are published or not. The documents may come from teaching and research institutions in France or abroad, or from public or private research centers.
L'archive ouverte pluridisciplinaire HAL, est destinée au dépôt et à la diffusion de documents scientifiques de niveau recherche, publiés ou non, émanant des établissements d'enseignement et de recherche français ou étrangers, des laboratoires publics ou privés. 


\section{Subtyping of Primary Aldosteronism in the AVIS-2 Study: Assessment of Selectivity and Lateralization}

\begin{abstract}
Giacomo Rossitto, ${ }^{1,21}$ Laurence Amar, ${ }^{2}$ Michel Azizi, ${ }^{2}$ Anna Riester, ${ }^{3}$ Martin Reincke, ${ }^{3}$ Christoph Degenhart, ${ }^{3}$ Jiri Widimsky $\mathrm{Jr}^{4}$ Mitsuhide Naruse, ${ }^{5}$ Jaap Deinum, ${ }^{6}$ Leo Schultzekool, ${ }^{6}$ Tomaz Kocjan, ${ }^{7}$ Aurelio Negro, ${ }^{8}$ Ermanno Rossi, ${ }^{8}$ Gregory Kline, ${ }^{9}$ Akiyo Tanabe, ${ }^{10}$ Fumitoshi Satoh, ${ }^{11}$ Lars Christian Rump, ${ }^{12}$ Oliver Vonend, ${ }^{12}$ Holger S. Willenberg, ${ }^{13}$ Peter Fuller, ${ }^{14}$ Jun Yang, ${ }^{14}$ Nicholas Yong Nian Chee, ${ }^{14}$ Steven B. Magill, ${ }^{15}$ Zulfiya Shafigullina, ${ }^{16}$ Marcus Quinkler, ${ }^{17}$ Anna Oliveras, ${ }^{18}$ Chin-Chen Chang, ${ }^{19}$ Vin Cent Wu ${ }^{19}$ Zusana Somloova, ${ }^{4}$ Giuseppe Maiolino, ${ }^{1}$ Giulio Barbiero, ${ }^{20}$ Michele Battistel, ${ }^{20}$ Livia Lenzini, ${ }^{1}$ Emilio Quaia, 20 Achille Cesare Pessina, ${ }^{1}$ and Gian Paolo Rossi ${ }^{1}$

${ }^{1}$ Department of Medicine-DIMED, University Hospital, Padova, Italy; ${ }^{2}$ Hypertension unit, Université de Paris, Inserm UMR970 and CIC1418, Hôpital Européen Georges Pompidou, F-75015 Paris, France; ${ }^{3}$ Medizinische Klinik und Poliklinik IV, Klinikum der Universität München, LMU München, München, Germany; ${ }^{4}$ 3rd Department of Medicine, Charles University Prague, General Hospital, Prague, Czech Republic; ${ }^{5}$ Department of Endocrinology, Clinical Research Institute, NHO Kyoto Medical Center and Endocrine Center, ljinkai Takeda General Hospital, Kyoto, Japan; ${ }^{6}$ Departments of Internal Medicine and Radiology, Radboud University Nijmegen, Nijmegen, The Netherlands; ${ }^{7}$ Department of Endocrinology, Diabetes and Metabolic Diseases, Faculty of Medicine, University of Ljubljana, Ljubljana, Slovenia; ${ }^{8}$ Department of Internal Medicine, Azienda Unità Sanitaria Locale, IRCCS Arcispedale S. Maria Nuova, Reggio Emilia, Italy; ${ }^{9}$ University of Calgary, Foothills Medical Centre, Calgary, Canada;

${ }^{10}$ Department of Diabetes, Endocrinology and Metabolism, National Center for Global Health and Medicine (NCGHM), Tokyo, Japan; ${ }^{11}$ Department of Nephrology, Tohoku University Hospital, Endocrinology and Vascular Medicine, Sendai, Japan; ${ }^{12}$ Department of Nephrology, Heinrich Heine University Düsseldorf, Düsseldorf, Germany; ${ }^{13}$ Division of Endocrinology and Metabolism, Rostock University Medical Center, Rostock, Germany; ${ }^{14}$ Department of Endocrinology, Monash Health, Clayton, Australia; ${ }^{15}$ Medical College of Wisconsin, Endocrinology Center, North Hills Health Center, Menomonee Falls, Wisconsin 53051; ${ }^{16}$ Department of Endocrinology, North-Western State Medical University named after I.I. Mechnikov, St. Petersburg, Russia; ${ }^{17}$ Endocrinology in Charlottenburg, 10627 Berlin, Germany; ${ }^{18}$ Nephrology Department, Hospital del Mar Universitat Autònoma de Barcelona, Barcelona, Spain; ${ }^{19}$ Department of Internal Medicine, National Taiwan University Hospital, Taipei, Taiwan; ${ }^{20}$ Institute of Radiology, University of Padova, Padova, Italy; and ${ }^{21}$ Institute of Cardiovascular and Medical Sciences, University of Glasgow, Glasgow, UK
\end{abstract}

ORCiD number: 0000-0002-7963-0931 (G.P. Rossi).; 0000-0002-9817-9875 (Martin Reincke).; 00000003-4620-4976 (Jun Yang).

Context: Adrenal venous sampling (AVS) is the key test for subtyping primary aldosteronism (PA), but its interpretation varies widely across referral centers and this can adversely affect the management of PA patients.

Objectives: To investigate in a real-life study the rate of bilateral success and identification of unilateral aldosteronism and their impact on blood pressure outcomes in PA subtyped by AVS.

Design and settings: In a retrospective analysis of the largest international registry of individual AVS data (AVIS-2 study), we investigated how different cut-off values of the selectivity index

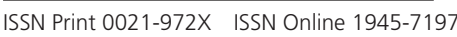

Printed in USA

(C) Endocrine Society 2019. All rights reserved. For permissions, please e-mail: journals. permissions@oup.com

Received 30 April 2019. Accepted 18 September 2019.

First Published Online 20 September 2019.

Corrected and Typeset 21 April 2020.
Abbreviations: APA, aldosterone-producing adenoma; AV, adrenal vein; AVIS, Adrenal Vein sampling International Study; AVS, adrenal venous sampling; IVC, inferior vena cava; LI, lateralization index; MRA, mineralocorticoid receptor antagonist; PA, primary aldosteronism; PAC, plasma aldosterone concentration; PASO, primary aldosteronism surgery outcome; PCC, plasma cortisol concentration; RASI, relative aldosterone secretion index; SI, selectivity index. 
(SI) and lateralization index (LI) affected rate of bilateral success, identification of unilateral aldosteronism, and blood pressure outcomes.

Results: AVIS-2 recruited 1625 individual AVS studies performed between 2000 and 2015 in 19 tertiary referral centers. Under unstimulated conditions, the rate of biochemically confirmed bilateral AVS success progressively decreased with increasing SI cut-offs; furthermore, with currently used LI cut-offs, the rate of identified unilateral PA leading to adrenalectomy was as low as $<25 \%$. A within-patient pairwise comparison of 402 AVS performed both under unstimulated and cosyntropin-stimulated conditions showed that cosyntropin increased the confirmed rate of bilateral selectivity for SI cut-offs $\geq 2.0$, but reduced lateralization rates $(P<0.001)$. Post-adrenalectomy outcomes were not improved by use of cosyntropin or more restrictive diagnostic criteria.

Conclusion: Commonly used SI and LI cut-offs are associated with disappointingly low rates of biochemically defined AVS success and identified unilateral PA. Evidence-based protocols entailing less restrictive interpretative cut-offs might optimize the clinical use of this costly and invasive test. (J Clin Endocrinol Metab 105: 2042-2052, 2020)

Key Words: aldosterone, aldosteronism, diagnosis, adrenal vein sampling, registry

$\mathbf{P}$ rimary aldosteronism (PA) is incorrectly regarded as a rare condition, despite evidence showing that it is the most common cause of endocrine hypertension (1-4). Failure to identify and subtype PA at an early stage leaves a multitude of patients exposed to life-long hyperaldosteronism, and thus to a high risk of cardiovascular events, particularly atrial fibrillation, as shown in both retrospective and prospective studies (5-8).

In the work-up of PA patients, the subtyping is a fundamental step, because patients with a unilateral form, mostly aldosterone-producing adenoma (APA) and unilateral adrenal hyperplasia $(9,10)$, benefit from laparoscopic adrenalectomy to obtain definitive correction of the hyperaldosteronism and often cure of arterial hypertension. Conversely, patients with bilateral PA, predominantly bilateral adrenal hyperplasia (also known as idiopathic hyperaldosteronism), require life-long medical treatment with a mineralocorticoid receptor antagonist (MRA), often in combination with multiple other antihypertensive agents.

To distinguish between unilateral and bilateral PA, all current guidelines advocate use of adrenal vein sampling (AVS) $(11,12)$, a technically demanding test where success is defined as bilateral selectivity, ie, adequate sampling of both adrenal veins. Confirmation of selectivity also serves to minimize the impact of two potential confounders when ascertaining lateralization of aldosterone excess: the degree of proximity of the catheter's tip to the adrenal cortex, and dilution effect from blood in accessory veins or inferior vena cava.

The criteria to define selectivity and lateralization remain variable, even at major tertiary centers where AVS is performed on a regular basis, as shown by data from a large international survey (AVIS-1) (13) and expert consensus reports $(14,15)$. This heterogeneity in interpretation can have a profound effect on the clinical decision-making, and thus on the usefulness of AVS.

The Adrenal Vein sampling International Study (AVIS)-2 was planned after completion of AVIS-1(13) with the aim of creating a large international registry of individual AVS data. The results of this study regarding patient outcomes, ie, correction of aldosteronism and rate of cured/improvement of arterial hypertension are reported elsewhere (16): not only did they provide a snapshot of what occurs in real-life and highlight the general outcome benefit of AVS-guided surgical decision-making but also demonstrated the inconsistencies in AVS use and their profound clinical implications (16). Based on those findings, in this study we explored the potential impact and usefulness of more standardized AVS interpretation criteria on management of PA patients. Hence, we herein report on: (i) the potential rate of selective (confirmed successful) AVS studies, (ii) the potential rate of unilateral PA suitable for adrenalectomy; (iii) the post adrenalectomy blood pressure outcomes as a function of the AVS protocol and of commonly advocated diagnostic cut-offs for the indexes defining selectivity (SI) and lateralization (LI).

\section{Methods}

The study rationale, design, center recruitment, inclusion/ exclusion criteria, population characteristics, and outcome analysis of AVIS-2 were reported in a separate paper (16) and are recapitulated in the Supplementary Methods; all supplementary material and figures are located in a digital research material repository (17). All procedures were carried out according to the Helsinki Declaration. The protocol of the study was approved by the Ethics Committee of both the coordinating center and the participating centers.

In brief, de-identified biochemical data from individual AVS studies were entered in a dedicated web-based platform 
(https://fm.dmcs.unipd.it) by local investigators who entered the units of measure as per local practice to avoid any conversion errors. The data collection form is shown in the Supplementary Material (17). Post hoc harmonization to conventional units was undertaken in the final database. After database locking, data were checked for internal consistency and standing queries were clarified with the lead investigators from the centers. AVS indices were defined as previously reported and per guidelines $(11,18,19)$ : (i) the selectivity index (SI) is the ratio between plasma cortisol concentration (PCC) in the adrenal vein (AV) and (infrarenal) inferior vena cava (IVC) and estimates the correct positioning of catheters in the adrenal vein $\left[\mathrm{PCC}_{\mathrm{AV}} / \mathrm{PCC}_{\mathrm{IVC}}\right]$; (ii) the "central/peripheral ratio", originally introduced by Espiner et al. (20) and renamed by some of us (G.R., G.P.R.) "relative aldosterone secretion index" (RASI) (21), is the ratio between the plasma aldosterone concentration (PAC) in each adrenal vein (AV) and inferior vena cava (IVC) divided by the degree of selectivity, ie, dilution, and identifies the contribution of the culprit (dominant) and non-culprit (non-dominant) adrenal to aldosterone secretion $\left[\left(\mathrm{PAC}_{\mathrm{AV}} / \mathrm{PCC}_{\mathrm{AV}}\right) /\left(\mathrm{PAC}_{\mathrm{IVC}} / \mathrm{PCC}_{\mathrm{IVC}}\right)\right]$; in the non-dominant side it is equivalent to the so-called "contralateral suppression index"; (iii) the Lateralization Index (LI) is the ratio between the higher and the lower RASI [or, after simplification: $\left(\mathrm{PAC}_{\text {dominant }} \mathrm{AV} / \mathrm{PCC}_{\text {dominant } \mathrm{AV}}\right) /\left(\mathrm{PAC}_{\text {non-dominant } \mathrm{AV}} /\right.$ $\left.\left.\mathrm{PCC}_{\text {non-dominant } \mathrm{AV}}\right)\right]$ and measures the imbalance in aldosterone secretion between the adrenal glands.

The blood pressure outcomes at follow-up were categorized according to predefined classes as cure, defined as normal blood pressure without any antihypertensive medication, no improvement, defined as lack of blood pressure reduction and/or need for increased number and/or dose of antihypertensive medications, and improvement, the last one split into marked and mild (Supplementary Table 1) (17). These classes are equivalent to those currently used (complete, partial, and absent clinical success) after publication of the clinical outcome (PASO) classification (22). Data were analyzed per protocol according to the original classification, but also by collapsing into a joint definition of partial clinical success the marked and mild improvement, in order to allow for a swift comparison with studies that used the PASO criteria and for a broad generalization of the conclusions.

The diagnostic and outcome impact of different interpretative rules for SI and LI on AVS data obtained with different AVS protocols (non-stimulated, ie, basal, or stimulated) was explored in the entire cohort. Cut-offs used for the analysis were those recommended by guidelines $(11,23)$ and/or endorsed by expert consensus documents $(14,15)$.

A paired, within-patient, within-AVS comparison was conducted for cases with available pre- and post-cosyntropin results. ROC (receiver operator characteristic) curve analyses were performed to assess the performance of SI interpretation rules obtained under unstimulated conditions (SI unstimulated) using the post-cosyntropin selectivity data, defined as a $\mathrm{SI}_{\text {cosyntropin }}$ cut-off $=5.0$, as reference standard. This was based on current use and evidence of a clear-cut bimodal separation after cosyntropin (please see results) (17). The Youden index was used to identify the optimal cut-off value for SI unstimulated, ie, the best combination of sensitivity and specificity using post-cosyntropin-ascertained selectivity as classification criterion. The value identified in this analysis (see Results) was included as one of the cut-offs for which the diagnostic/outcome performance was explored.
A sensitivity analysis of the impact of cosyntropin was also performed by repeating the paired comparisons and excluding each individual center stepwise.

The values of SI, LI, and RASI, which showed a skewed distribution at the Kolmogorov-Smirnov test, are reported as median and interquartile range (IQR) and compared across groups with non-parametric Wilcoxon test. The frequency of categorical variables was analyzed with Pearson's $\chi^{2}$; McNemar's test was used for comparison between different diagnostic criteria within the same population. Significance was set at $P<0.05$. SPSS for Mac (vers. 25 for Mac, IBM-SPSS Bologna, Italy), GraphPad, Prism (vers. 8.1.1 for Mac, GraphPad Software, La Jolla, CA, USA), and MedCalc (MedCalc Software Ostend Belgium, vers. 15.8) software were used for the statistical analysis.

\section{Results}

\section{Study population}

The whole database included 1820 individual AVS datasets from consecutive patients studied in 19 centers (Supplementary Table 2) (17). However, to focus upon current AVS practice, the oldest AVS datasets were excluded and the analysis was limited to 1625 individual cases performed from 2000 to 2015 (Fig. 1). The clinical/demographic features of the PA patient population were reported in detail elsewhere (16) and are recapitulated in the Supplementary Table 3 (17).

Overall, AVS datasets were available in $1274 \mathrm{pa}-$ tients under unstimulated conditions and in 865 cases after pharmacologic stimulation (742 with cosyntropin [85.8\%], and 123 with metoclopramide). In 402 patients $(24.7 \%$ of the total $)$ both unstimulated and cosyntropin-stimulated AVS was performed during the same procedure. This furnished the opportunity for a paired within-patient-within-AVS comparison. Metoclopramide was only used in one center, as already described $(19,24)$; the results obtained with metoclopramide are reported here simply for comparison with the cosyntropin-stimulated AVS.

In 1004/1274 of AVS performed under unstimulated conditions, in 637/742 after stimulation and in 317/402 AVS after both unstimulated and cosyntropinstimulated conditions, post-AVS outcome data were available.

\section{Rate of confirmed, successful AVS selectivity at different biochemical SI cut-off definitions}

Unstimulated AVS. The analysis of the success rate of adrenal catheterization under unstimulated conditions showed that increasingly higher biochemical definitions of the SI cut-off up to 5.0 resulted in a progressive fall in the rate of confirmed selective studies on each side and bilaterally (Fig. 2 and Supplementary Table 4) (17). With the most 




Figure 1. Study population. The flowchart illustrates the distribution of the patients across AVS protocols. In grey, AVS performed under both unstimulated and cosyntropin-stimulated conditions. Abbreviation: AVS, adrenal venous sampling.

stringent cut-off of 5.0 , only $38.3 \%$ of the studies were deemed bilaterally selective; however, the rate rose to $52.4 \%$ and $67.3 \%$ with the commonly used cut-off values of 3.0 and 2.0, respectively.

Post-cosyntropin stimulated AVS. In contrast to the drop in confirmed success observed with increasingly restrictive SI cut-off definitions under unstimulated conditions or during metoclopramide stimulation (Fig. 2 and Supplementary Fig. 1) (17), use of more stringent SI cut-off definitions had less impact on catheterization success when measured after cosyntropin infusion. Despite more stringent interpretation criteria, the proportion of AVS deemed successful decreased only by $9 \%$, ie, from $90.2 \%$ at an SI cut-off of 1.1 , to $81.3 \%$ at 5.0 .

Importantly, the currently recommended SI cut-off of 5.0 for cosyntropin-stimulated AVS $(11,15)$ was associated with a higher rate of bilaterally successful AVS procedures than any SI cut-off definitions $\geq 2.0$ under unstimulated conditions (Fig. 2 and Supplementary Table 4) (17).

Use of intraprocedural cortisol assay. The intraprocedural rapid cortisol assay (IRCA) was used to confirm AVS selectivity under unstimulated conditions only in two centers for a total of 178 patients; it was associated with higher rates of selectivity at each SI cutoff value (Supplementary Table 5) (17).
Comparison of the IRCA cohort with cosyntropinstimulated AVS showed a similar rate of bilateral selectivity for low SI cut-offs and a better performance of the latter only at SI cut-off values $\geq 3.0$ (Supplementary Table 5) (17).

\section{Comparison of unstimulated vs stimulated AVS: determination of the optimal unstimulated SI cut-off}

The within-patient-within-AVS pairwise comparison of unstimulated and post-cosyntropin data confirmed increased values of the SI and rate of bilateral selectivity after stimulation (Fig. 3). Notably, the SI values after cosyntropin (Supplementary Fig. 2) showed a bimodal distribution, ie, a clear separation of biochemically successful and non-successful studies, demarcated by the post-cosyntropin SI cut-off definition of 5.0, already in common use $(11,15)$.

We used this value as reference for sampling success in a ROC curve analysis to explore the diagnostic performance of unstimulated SI values in this cohort. In general, SI values calculated from unstimulated measurements provided a reasonable accuracy in defining catheterization success (AUC 0.756 (0.724-0.785), $P<0.0001$ vs the identity line AUC of 0.50$)$. The Youden Index value of 1.4 from unstimulated measurements generated the highest combined accuracy, with $92 \%$ sensitivity and $62 \%$ specificity (Fig. 3, Supplementary 


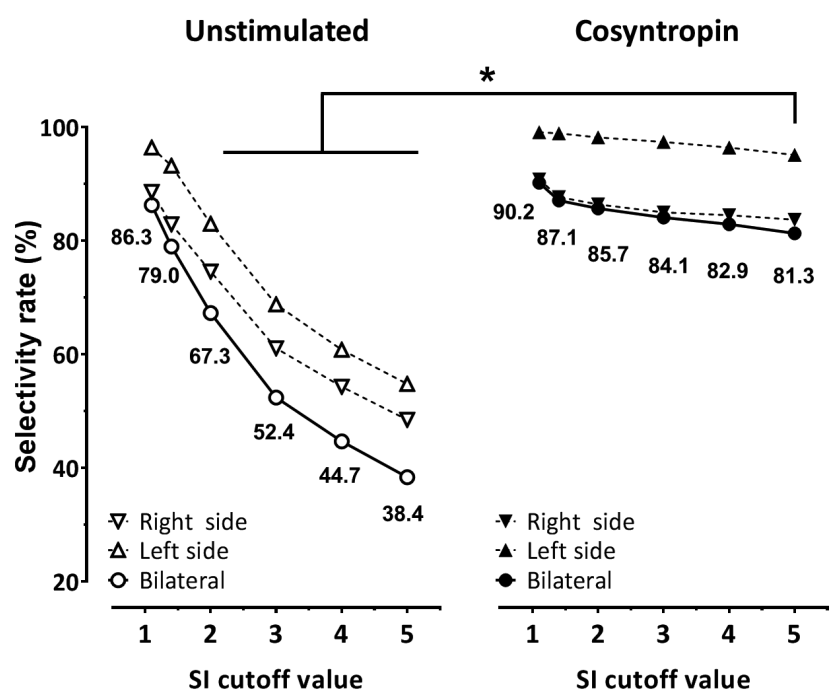

Figure 2. Rate of selectivity as a function of different $\mathrm{SI}$ values (definitions). The left panel (empty symbols) shows results under unstimulated conditions ( $n=1274)$; the right panel (closed symbols) shows cosyntropin-stimulated AVS $(n=742)$ results. Use of increasingly stringent SI cut-off definitions resulted in a progressive fall in the rate of diagnostic studies under unstimulated conditions, but to a much lower extent under cosyntropin stimulation. Please note that a definition of selectivity by $\mathrm{SI}_{\text {cosyntropin }} \geq 5.0$ performed better

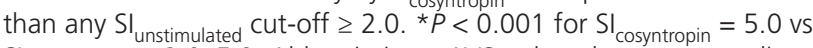
$\mathrm{SI}_{\text {unstimulated }}=2.0-5.0$. Abbreviations: AVS, adrenal venous sampling; $\mathrm{LI}$, lateralization index; SI, selectivity index;

Table 6) (17). When applied to the entire AVIS-2 database this definition offered a rate of successful selectivity under unstimulated conditions similar to that of a postcosyntropin SI $\geq 5.0$, regardless of IRCA being used or not (Fig. 2, Supplementary Table 5) (17). Results were identical when we used a post-cosyntropin SI cut-off definition of 4.0 (15).

\section{Impact of biochemical diagnostic indices on the diagnosis of unilateral disease and adrenalectomy} Identification of unilateral or bilateral aldosterone excess is possible only after confirmation of technical AVS success according to SI measures. We therefore assessed the rate of patients who could be deemed to have unilateral disease, defined by LI cut-off definitions ranging from 2.0 to $5.0(11,14,15)$, in concert with SI cut-off definitions of 1.4, 2.0, and 3.0 for unstimulated measurements and 5.0 for postcosyntropin values. This showed that the rate of lateralization among bilaterally successful studies, dropped significantly with adoption of higher SI cut-off definitions and with each unit increase in LI cut-off definition (Fig. 4, top; Supplementary Table 7 and Supplementary Fig. 3) (17). With commonly used biochemical definitions under unstimulated and cosyntropin-stimulated conditions, ie, 'SI $\geq 2.0+\mathrm{LI} \geq 3.0$ ' and ' $\mathrm{SI} \geq 5.0+\mathrm{LI} \geq 4.0$ ', the proportion of patients deemed to have unilateral disease was $39.8 \%$ and $36.6 \%$, respectively $(P=0.174$ for comparison). It increased to $55.6 \%(P<0.001)$ with less stringent definitions of unstimulated SI and/or LI, ie, a SI $\geq 1.4$ definition for selectivity combined with a cut-off of 2.0 for lateralization.

Importantly, there was a lower rate of patients submitted to adrenalectomy after application of more

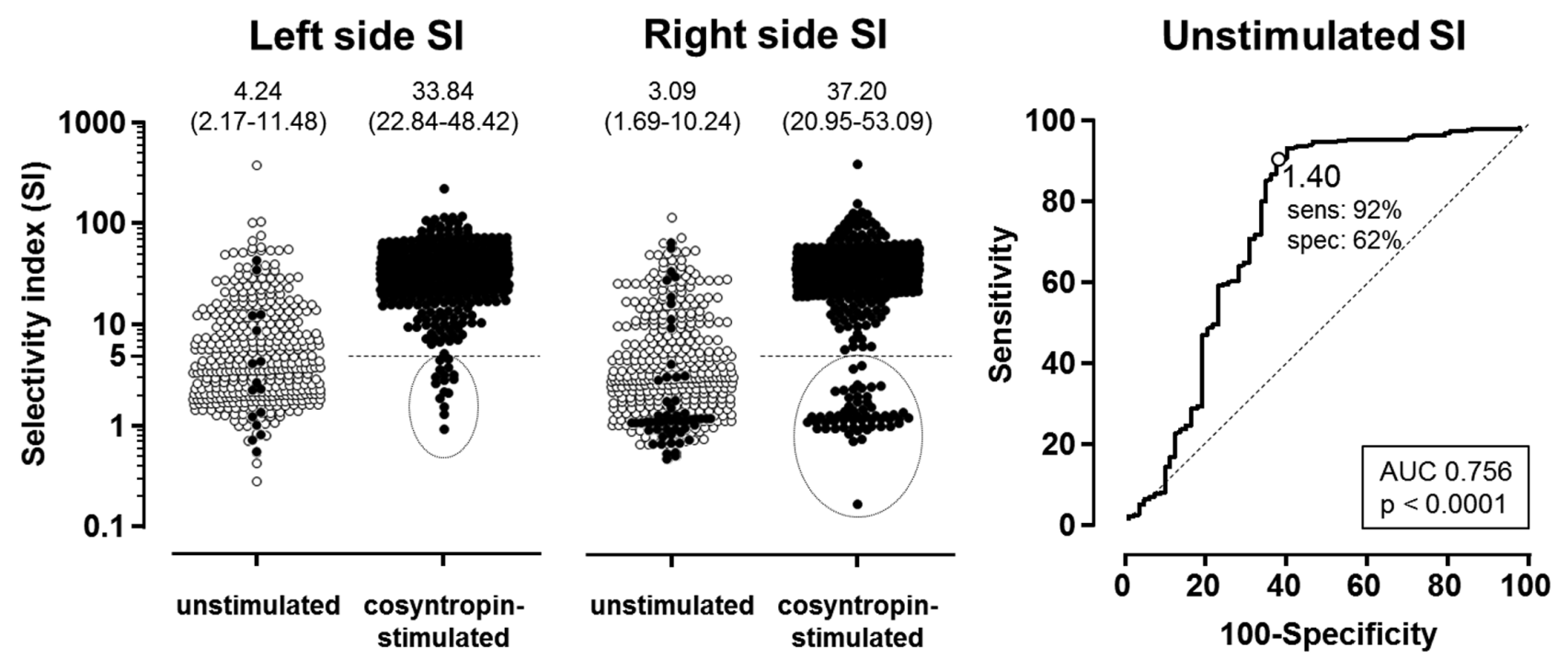

Figure 3. Impact of cosyntropin on SI and identification of optimal unstimulated SI. The left panel shows paired comparison of SI values in the left and right side obtained under unstimulated and post-cosyntropin conditions in 402 patients who were submitted to unstimulated and cosyntropin- stimulated AVS during the same procedure. Medians and interquartile ranges are shown on top. Closed symbols in the unstimulated plots correspond to values that were judged to be unsuccessful, ie, below $<5.0$, post-cosyntropin. Right panel: ROC curve of unstimulated SI values performed using as golden reference a post-cosyntropin $\mathrm{SI} \geq 5.0$. Please note that the optimal cut-off for unstimulated $\mathrm{SI}$, ie, Youden index, resulted to be 1.4 , which corresponded to a $92 \%$ sensitivity and a $62 \%$ specificity. Abbreviations: AUC, area under the curve; ROC, receiver operating characteristic; $\mathrm{SI}$, selectivity index. 


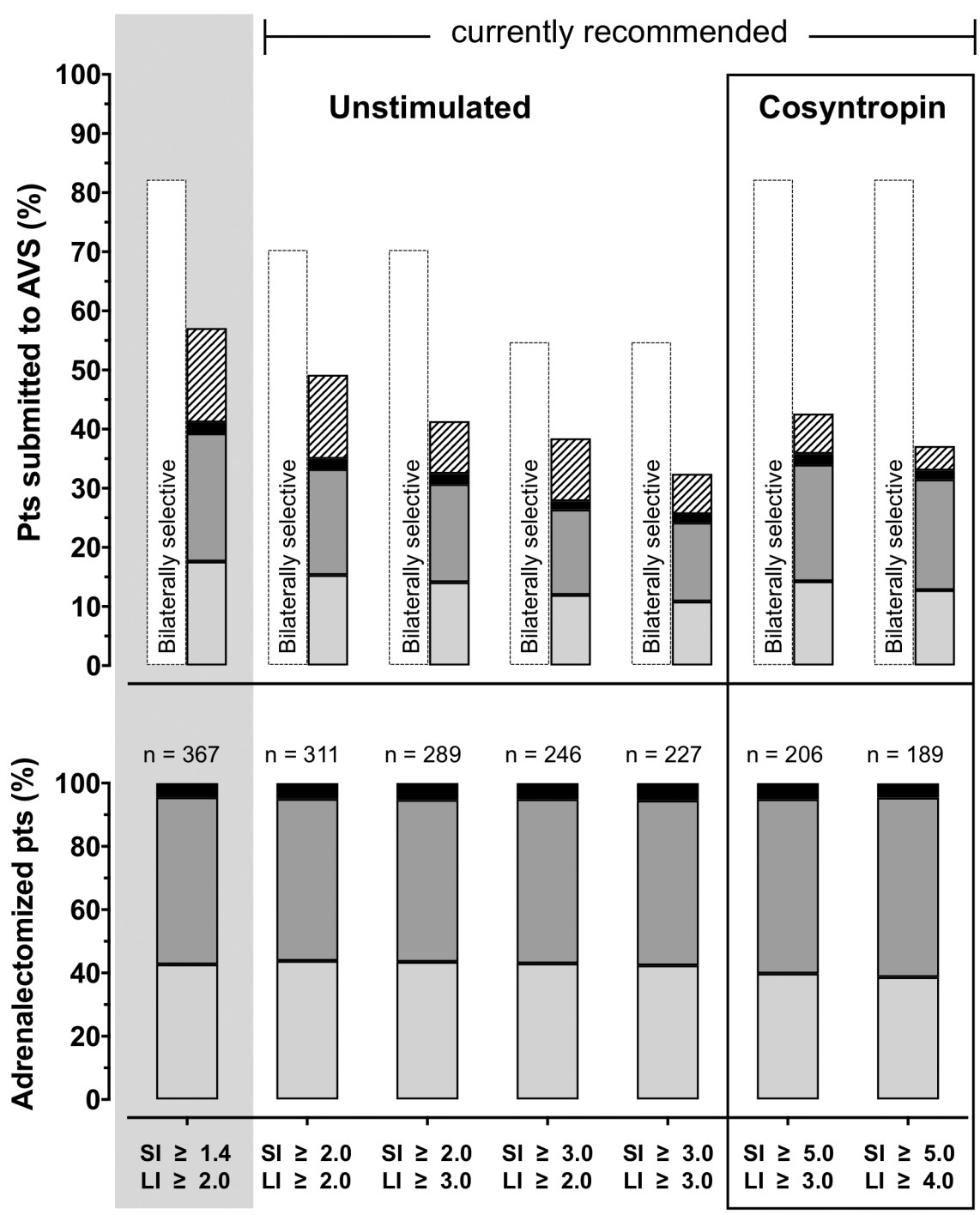

\section{Cure $\square$ Improvement $\square$ No improvement $\mathbb{2}$ No adrenalectomy}

Figure 4. Lateralization, adrenalectomy and blood pressure outcomes according to different sets of diagnostic criteria. Top panel: rate of bilaterally selective (empty bars), no adrenalectomy (dashed bars) and adrenalectomy with ensuing outcomes in the all patients referred to AVS under unstimulated and cosyntropin-stimulated conditions with available clinical outcome data ( $n=880 / 1004$ and 580/637, respectively), as a function of set of diagnostic criteria. Those currently recommended are in the mid and right part of the top panel, while a more lenient set (SI > 1.40 and $\mathrm{LI}>2.0$ ) based on the above paired-analysis results is shown at the extreme left (grey box). Filled bars indicate adrenalectomized patients, with rates of post-surgical cure, improvement, and no improvement in shades of grey (legend at bottom). The rate of patients fulfilling the same set of homogeneous criteria for selectivity and lateralization but not submitted to surgery are indicated by the superimposed dashed bars. Please note the similar rate of bilateral selectivity at an unstimulated $\mathrm{SI}>1.4$ and at a post-cosyntropin $\mathrm{SI}>5.0$, but the lower rate of ascertained lateralization and adrenalectomy if a post-cosyntropin $\mathrm{SI}>5.0$ were used. Bottom panel: The rate of cure, improvement and no improvement among patients who underwent adrenalectomy meeting the sets of criteria described above, ie, the filled bars from top panel, were similar across sets of criteria. This indicates that use of more restrictive definitions of success and unilateral disease is associated with more patients denied potentially curative surgery (absolute numbers shown on top of each bar) but no better outcomes.

stringent definitions for unilateral disease, to a nadir of less than $25 \%$ with an interpretative SI $\geq 3.0$ and LI $\geq 4.0$ (Supplementary Table 7) (17). However, the proportion of patients referred for adrenalectomy among those with AVS evidence of unilateral disease was higher with more restrictive criteria for lateralization, suggesting more physicians' confidence in results that meet the stricter definitions (Fig. 4, top, and Supplementary Table 7) (17).

\section{Impact of cosyntropin use upon the} determination of unilateral disease

A pairwise comparison of the bilaterally successful AVS under both unstimulated and cosyntropin-stimulated conditions showed a highly significant $(P<0.001)$ decrease of the LI values between baseline and post cosyntropin, a finding confirmed in the subcohort of those who had a unilateral form of PA unambiguously established by biochemical cure at follow-up post 
adrenalectomy ( $\mathrm{n}=149 / 402)$. Hence, after cosyntropin stimulation, notwithstanding the higher rate of bilaterally successful AVS studies, a lower proportion of patients was judged to have a unilateral form of PA (Fig. 4 top panel and Supplementary Table 7) (17).

By calculating the relative aldosterone secretion (RASI) to assess the contribution of the culprit and nonculprit adrenal to the LI value (19-21), we could clarify that the LI decreased because of a more prominent drop of RASI in the dominant than the nondominant side (Table 1). This fall was specific to cosyntropin, as it did not occur during metoclopramide stimulation (Supplementary Fig. 4) (17). A sensitivity analysis performed by stepwise elimination of each individual center showed similar results (Supplementary Table 8) (17).

In line with these findings and regardless of the LI definition used, the number of cases judged to be unilateral post cosyntropin decreased significantly compared with the rate determined by unstimulated AVS measures (Table 2). Accordingly, the proportion of PA patients with lateralized AVS results under unstimulated conditions and post-cosyntropin AVS results indicating bilateral disease increased and could exceed $30 \%$ depending on the diagnostic criteria used (grey shaded cells in Table 2).

\section{Impact of diagnostic cut-off definitions on clinical management and blood pressure outcome}

The rate of patients referred to surgery decreased in accordance with the propensity of individual centers to trust more restrictive LI cut-off definitions of unilateral disease or results from cosyntropin-stimulated AVS (Fig. 4, top). However, the relative distribution of blood pressure outcomes (cure, improvement, and no improvement) was remarkably similar despite different diagnostic criteria or protocols used (Fig. 4 bottom panel; Supplementary Table 9) (17). A borderline-significant shift from mild to marked within the class improvement was observed with cosyntropin. However, this occurred at the cost of a much lower rate of adrenalectomies out of the selective (successful) AVS studies. Importantly, in the "paired measurement" cohort with unilateral disease according to unstimulated conditions, but no lateralization post-cosyntropin, only a minority of the patients received adrenalectomy (Supplementary Table 10) (17), possibly due to physicians' perceived diagnostic uncertainty. While this limited the statistical power of this post-adrenalectomy outcome subanalysis, it is worth emphasizing that all such patients showed at least improvement.

\section{Discussion}

Thus far, the performance of AVS protocols and interpretations for defining procedural success and lateralization has only been examined in relatively small PA cohorts with limited geographical representation. We have now reported the performance of widely used SI and LI cut-off definitions and AVS protocols for the identification of unilateral forms of PA in the largest international registry of individual AVS studies. These results confirm and explain, at least in part, the disappointingly low rates of surgical cure of hypertension obtained in the AVIS-2 study by using local and stringent criteria for the interpretation of AVS in real-life clinical practice (16).

By examining the selectivity rate as a function of the SI cut-off definitions recommended by expert consensus under unstimulated conditions $(14,15)$ we report that approximately one-third of PA patients submitted to AVS would not have gained any diagnostic benefit: even with a more lenient SI cut-off definition of 2.0 approximately one third of AVS were judged to be non-selective (Fig. 2). With a more stringent definition, originally endorsed by the 2008 Endocrine Society guidelines (23)

Table 1. Paired comparison of relative aldosterone secretion index (RASI) by side and lateralization index (LI) between unstimulated and post-cosyntropin conditions

\begin{tabular}{|c|c|c|c|c|c|c|c|c|}
\hline \multirow[b]{2}{*}{ Indexes } & \multicolumn{4}{|c|}{ Whole cohort } & \multicolumn{4}{|c|}{ Unilateral PA } \\
\hline & Unstimulated & $\begin{array}{c}\text { Post } \\
\text { cosyntropin }\end{array}$ & $\begin{array}{l}\% \Delta \text { RASI } \\
\text { (median) }\end{array}$ & $P$ & Unstimulated & $\begin{array}{c}\text { Post } \\
\text { cosyntropin }\end{array}$ & $\begin{array}{l}\% \Delta \text { RASI } \\
\text { (median) }\end{array}$ & $P$ \\
\hline \multicolumn{9}{|l|}{ RASI } \\
\hline Dominant side & $\begin{array}{c}7.26 \\
(2.79-13.00)\end{array}$ & $\begin{array}{c}3.93 \\
(2.71-5.32)\end{array}$ & $-42.0 \%$ & $<0.001$ & $\begin{array}{c}8.41 \\
(5.88-10.90)\end{array}$ & $\begin{array}{c}4.25 \\
(2.76-6.69)\end{array}$ & $-93.2 \%$ & $<0.001$ \\
\hline $\begin{array}{l}\text { Non-dominant } \\
\text { side }\end{array}$ & $\begin{array}{c}1.00 \\
(0.47-2.34)\end{array}$ & $\begin{array}{c}1.25 \\
(0.34-2.33)\end{array}$ & $-2.6 \%$ & ns & $\begin{array}{c}0.52 \\
(0.26-1.04)\end{array}$ & $\begin{array}{c}0.37 \\
(0.19-0.89)\end{array}$ & $-2.5 \%$ & 0.020 \\
\hline 니* & $\begin{array}{c}3.81 \\
(1.73-20.40)\end{array}$ & $\begin{array}{c}2.52 \\
(1.41-10.80)\end{array}$ & - & $<0.001$ & $\begin{array}{c}17.71 \\
(3.87-33.28)\end{array}$ & $\begin{array}{c}9.43 \\
(3.18-19.84)\end{array}$ & - & $<0.001$ \\
\hline
\end{tabular}

Paired comparison in the subcohort of patients with bilaterally selective AVS on both non-stimulated (unstimulated) and post cosyntropin conditions $(\mathrm{n}=402) . \mathrm{PA}=$ primary aldosteronism. Data presented as median (interquatile range). ${ }^{*} \mathrm{SI} \geq 2.0$ was used for definition of unstimulated $\mathrm{LI}$; $\mathrm{SI} \geq 5.0$ was used for definition of post-cosyntropin LI. Results are consistent across different SI cut-off values (not shown). Wilcoxon test, significance set at $P<0.05$. 


\section{Table 2. Diagnostic discrepancy between paired unstimulated and cosyntropin-stimulated AVS results}

Cosyntropin-stimulated

\begin{tabular}{|c|c|c|c|c|c|c|c|c|c|}
\hline & & & & \multirow{2}{*}{\multicolumn{6}{|c|}{$\mathrm{SI} \geq \mathbf{5 . 0}$}} \\
\hline & & & & & & & & & \\
\hline & & & & \multicolumn{2}{|c|}{$\mathrm{LI} \geq \mathbf{3 . 0}$} & \multirow[b]{2}{*}{ P McNemar } & \multicolumn{2}{|c|}{$\mathrm{LI} \geq 4.0$} & \multirow[b]{2}{*}{ P McNemar } \\
\hline & & & & Lat & Bilat & & Lat & Bilat & \\
\hline \multirow[t]{16}{*}{ Unstimulated } & \multirow[t]{5}{*}{$\mathrm{SI} \geq 1.4$} & $\mathrm{LI} \geq 2.0$ & Lat & $118(43.9)$ & $74(27.5)$ & $<0.001$ & $106(39.4)$ & $86(32.0)$ & $<0.001$ \\
\hline & & $\mathrm{LI} \geq 3.0$ & Lat & $112(41.6)$ & $43(16)$ & 0.002 & $102(37.9)$ & $53(19.7)$ & $<0.001$ \\
\hline & & & Bilat & $18(6.7)$ & $96(35.7)$ & & $10(3.7)$ & $104(38.7)$ & \\
\hline & & $\mathrm{LI} \geq 4.0$ & Lat & - & - & - & $91(33.8)$ & $38(14.1)$ & 0.037 \\
\hline & & & Bilat & - & - & & $21(7.8)$ & $119(44.2)$ & \\
\hline & \multirow[t]{6}{*}{$\mathrm{SI} \geq 2.0$} & $\mathrm{LI} \geq 2.0$ & Lat & $93(42.3)$ & $65(29.5)$ & $<0.001$ & $83(37.7)$ & 75 (34.1) & $<0.001$ \\
\hline & & & Bilat & $8(3.6)$ & $54(24.5)$ & & $3(1.4)$ & $59(26.8)$ & \\
\hline & & $\mathrm{LI} \geq 3.0$ & Lat & $88(40.0)$ & $39(17.7)$ & 0.001 & $80(36.4)$ & $47(21.4)$ & $<0.001$ \\
\hline & & & Bilat & $13(5.9)$ & $80(36.4)$ & & $6(2.7)$ & 87 (39.5) & \\
\hline & & $\mathrm{LI} \geq 4.0$ & Lat & - & - & - & $71(32.3)$ & $32(14.5)$ & 0.020 \\
\hline & & & Bilat & - & - & & $15(6.8)$ & $102(46.4)$ & \\
\hline & \multirow[t]{5}{*}{$\mathrm{SI} \geq 3.0$} & $\mathrm{LI} \geq 2.0$ & $\begin{array}{l}\text { Lat } \\
\text { Bilat }\end{array}$ & $\begin{array}{c}68(44.4) \\
4(26)\end{array}$ & $\begin{array}{l}42(27.5) \\
39(255)\end{array}$ & $<0.001$ & $62(40.5)$ & $\begin{array}{l}48(31.4) \\
41(26.8)\end{array}$ & $<0.001$ \\
\hline & & $\mathrm{LI} \geq 3.0$ & Lat & $64(41.8)$ & $25(16.3)$ & 0.005 & $59(38.6)$ & $30(19.6)$ & $<0.001$ \\
\hline & & & Bilat & $8(5.2)$ & $56(36.6)$ & & $5(3.3)$ & $59(38.6)$ & \\
\hline & & $\mathrm{LI} \geq 4.0$ & Lat & - & & & $52(34.0)$ & $19(12.4)$ & ns \\
\hline & & & Bilat & - & & & $12(7.8)$ & $70(45.8)$ & \\
\hline
\end{tabular}

Paired diagnostic comparison in the subcohort of patients with bilaterally selective AVS on both unstimulated and post cosyntropin conditions $(n=402)$. Data presented as $n(\%)$, according to use of different diagnostic criteria. Lat = AVS results suggesting lateralization according to corresponding diagnostic cut-off values; Bilat = AVS results suggesting bilateral PA according to corresponding diagnostic cut-off values. Baseline LI cut-off $\geq 4.0$ was not compared with post cosyntopin $\mathrm{LI}$ cut-off $\geq 3.0$ because there is no published evidence of any center using a more stringent approach under unstimulated conditions. McNemar test, significance set at $P<0.05$. Abbreviations: AVS, adrenal venous sampling; LI, lateralization index; ns, not significant; SI, selectivity index.

and still used in many centers (15), this rate of "failed" AVS was even higher, confirming previous observations from a smaller study (18).

The second important finding relates to the use of use of cosyntropin (synthetic adrenocorticotropic hormone) stimulation, which has become popular by virtue of its ability to generate higher SI values, thus confirming technical success in sampling. Cosyntropin can maximize the step-up of cortisol between the inferior vena cava (a surrogate for cortisol concentration in peripheral blood) and each adrenal vein. Moreover, it minimizes stress-induced steroid fluctuations and any factitious gradients during sequential (nonsimultaneous) AVS. The present results confirmed that cosyntropin infusion can increase the apparent AVS success rate when using very stringent SI cut-off definitions (Supplementary Figs 1, 4) (17); the known secretagogue effect of cosyntropin on cortisol and the selective secretagogue effect of metoclopramide on aldosterone (21) can explain these findings, as suggested also by previous single-center studies $(18,25)$.

More importantly, the AVIS-2 registry comprised the largest collection of AVS studies performed under both unstimulated and cosyntropin-stimulated conditions in the same patient during the same AVS procedure. As such, it offered a unique opportunity for a paired comparison of both selectivity and lateralization rates achieved with the two protocols. This within-patient and within-AVS analysis revealed a bimodal distribution of SI values post-cosyntropin, with a discriminating level corresponding to the commonly used post-cosyntropin SI cut-off definition of 5.0 (Fig. 3 and Supplementary Fig. 2) (17). Thus, even after stimulation and notwithstanding the aforementioned effect of cosyntropin on the SI, a subset of patients still had non-successful results, indicating that cosyntropin stimulation cannot resolve true catheterization failure due to inadequate catheter's positioning and/or unfavorable adrenal vein anatomy. The latter is not rare, as it has been documented in approximately $15 \%$ of PA patients $(26,27)$.

By using the post-cosyntropin selectivity as a reference, we found that the unstimulated SI definition that offered the best combination of sensitivity and specificity was $\geq 1.4$ (Fig. 3, Suppl. Table 6) (17). This definition corresponds to a rate of bilaterally selective cases similar to that achieved using the post-cosyntropin SI $\geq 5.0$ or $\geq 4.0$. These findings, along with a substantial loss of diagnostically usable AVS studies (Fig. 2) represent, in our view, a compelling argument against use of stringent SI definitions when measured under unstimulated conditions. The proposal of using less restrictive 
definitions is also supported by the lack of improvement in clinical outcomes with the use of more restrictive criteria (Fig. 4).

The third main finding of the study was that only a minority of the PA patients submitted to AVS (from one fifth to one third, depending on the combination of currently recommended restrictive SI and LI cut-off definitions) could be eventually referred for unilateral adrenalectomy with currently recommended SI and LI criteria. This low rate is consistent with the rate of AVSguided adrenalectomy seen in AVIS-2 (31.9\%), when the diagnosis was based on the criteria used at each center (16).

Overall, the low rates of biochemically defined apparent success, unilateral diagnoses, and referral for surgery may question the usefulness of a test that, besides being costly and invasive, is supposed to identify candidates for adrenalectomy. However, by applying an estimate of bypertension cure rate equal to that seen in the subset of patients who received AVS-guided adrenalectomy in AVIS-2 (16), ie, 40\%, to all those identified as likely affected by a unilateral form following less restrictive criteria (Fig. 3), the rate of patients cured based on AVS results would double, from 1 in every 8 (16) to approximately 1 in every 4 .

The increased rate of bilateral catheterization success provided by cosyntropin in the majority, albeit not all patients, could not resolve this disappointingly low diagnostic AVS "yield", in that the serious drawback of cosyntropin was a consistent drop in the lateralization index, as a result of a greater decrease of the relative aldosterone secretion index in the culprit than the contralateral side (Table 1). This drop, already observed in smaller studies $(25,28)$, may have led to judging up to $32 \%$ of PA patients as having a bilateral disease despite evidence of lateralization under unstimulated conditions. It may also justify the borderline-significant shift from mild to marked improvement observed with cosyntropin and likely due to a preselection of the most florid phenotypes. Unfortunately, the higher rate of adrenalectomy among patients identified as unilateral PA based on cosyntropin stimulation rather than under unstimulated sampling suggests that clinicians used to put higher confidence in post-cosyntropin measures. On the contrary, our data conclusively disprove the contention that cosyntropin stimulation would increase the accuracy of AVS for identifying unilateral PA by maximizing the secretion of aldosterone from an APA (or other unilateral forms of PA), one of the original premises for using cosyntropin: overall, cosyntropin did not result in higher rates of cure or better hard outcomes (Fig. 4).
Some limitations must be acknowledged along with the strengths of the AVIS-2 study, as discussed elsewhere (16). It should be acknowledged that, unlike for the surgically curable unilateral PA, there is still no gold standard for the diagnosis of bilateral adrenal hyperplasia. Therefore, to standardize AVS performance, we had to explore the clinical outcomes associated with currently recommended AVS diagnostic criteria and to compare them with sets of criteria homogeneously applied to the entire cohort, regardless of center-specific preferences.

It might be argued that the observational design and lack of predefined criteria to establish definitions of AVS success and lateralization, which were left to participating centers, can be main limitations of AVIS2. In truth, these can represent major strengths of this study, as they allowed gathering information of the use of AVS in real-life clinical practice. Additional considerable strengths are a predefined protocol for prospective data acquisition and the comprehensive collection of individual biochemical data from the largest worldwide multicenter cohort of subtyped PA patients.

In summary, this study showed that the full diagnostic potential of AVS is largely unrealized, even in major referral centers with research expertise. The use of cosyntropin during AVS, while increasing the rate of studies judged to be successful, resulted in decreased lateralization, thus masking true unilateral disease in a considerable proportion of cases. It is likely that cosyntropin allows identification of only the most severe PA phenotypes (21), thus explaining why it did not prove superior to a simpler computed tomography scan-only approach in an outcome-based randomized trial (29). Hence, based on data from the largest collection of AVS results generated from around the world, we recommend unstimulated AVS as the optimal AVS protocol, together with the adoption of more lenient diagnostic criteria (eg, SI > 1.4) in concert with expert clinical assessment and other predictors of unilateral disease. The use of the rapid intraprocedural assays and/or of markers of selectivity that have a higher adrenal-to-peripheral blood step-up than cortisol, such as metanephrines or androstenedione (30-32), can be promising strategies to improve the clinical yield of AVS and subsequent patient outcomes.

\section{Acknowledgments}

Financial Support: This study was supported in part by research grants to G.P.R. from FORICA (The Foundation for advanced Research In Hypertension and CArdiovascular diseases) and the Società Italiana dell'Ipertensione 
Arteriosa; from the Else Kröner-Fresenius-Stiftung to M.R., A.R., M.R. and J.D. received support from the Deutsche Forschungsgemeinschaft (DGE, German Research Foundation, Projektnummer 314061271-TRR 205'.

Clinical Trial Information: The AVIS was registered at clinicaltrials.gov number NCT01234220.

\section{Additional Information}

Correspondence: Gian Paolo Rossi, MD. FACC, FAHA., Hypertension Unit - Department of Medicine -DIMED, University Hospital via Giustiniani, 2. 35126 Padova, Italy. E-mail: gianpaolo.rossi@unipd.it

Disclosure Summary: There is no conflict of interest or relationship with industry to be disclosed. All authors have read and approved the manuscript.

Data Availability: Restrictions apply to the availability of data generated or analyzed during this study to preserve patient confidentiality. The corresponding author will on request detail the restrictions and any conditions under which access to data may be provided.

\section{References}

1. Rossi GP, Bernini G, Caliumi C, Desideri G, Fabris B, Ferri C, Ganzaroli C, Giacchetti G, Letizia C, Maccario M, Mallamaci F, Mannelli M, Mattarello MJ, Moretti A, Palumbo G, Parenti G, Porteri E, Semplicini A, Rizzoni D, Rossi E, Boscaro M, Pessina AC, Mantero F. A prospective study of the prevalence of primary aldosteronism in 1,125 hypertensive patients. J Am Coll Cardiol. 2006;48(11):2293-2300.

2. Douma S, Petidis K, Doumas M, Papaefthimiou P, Triantafyllou A, Kartali N, Papadopoulos N, Vogiatzis K, Zamboulis C. Prevalence of primary hyperaldosteronism in resistant hypertension: a retrospective observational study. Lancet 2008;371(9628):1921-1926.

3. Monticone S, Burrello J, Tizzani D, Bertello C, Viola A, Buffolo F, Gabetti L, Mengozzi G, Williams TA, Rabbia F, Veglio F, Mulatero P. Prevalence and clinical manifestations of primary aldosteronism encountered in primary care practice. J Am Coll Cardiol. 2017;69(14):1811-1820.

4. Mulatero P, Monticone S, Burrello J, Veglio F, Williams TA, Funder J. Guidelines for primary aldosteronism: uptake by primary care physicians in Europe. $J$ hypertens 2016;34(11):2253-2257.

5. Milliez P, Girerd X, Plouin PF, Blacher J, Safar ME, Mourad JJ. Evidence for an increased rate of cardiovascular events in patients with primary aldosteronism. J Am Coll Cardiol. 2005;45(8):1243-1248.

6. Rossi GP, Maiolino G, Flego A, Belfiore A, Bernini G, Fabris B, Ferri C, Giacchetti G, Letizia C, Maccario M, Mallamaci F, Muiesan ML, Mannelli M, Negro A, Palumbo G, Parenti G, Rossi E, Mantero F. Adrenalectomy lowers incident atrial fibrillation in primary aldosteronism patients at long term. Hypertension 2018;71(4):585-591.

7. Hundemer GL, Curhan GC, Yozamp N, Wang M, Vaidya A. Cardiometabolic outcomes and mortality in medically treated primary aldosteronism: a retrospective cohort study. Lancet Diabetes Endocrinol. 2018;6(1):51-59.

8. Monticone S, D’Ascenzo F, Moretti C, Williams TA, Veglio F, Gaita F, Mulatero P. Cardiovascular events and target organ damage in primary aldosteronism compared with essential hypertension: a systematic review and meta-analysis. Lancet Diabetes Endocrinol. 2018;6(1):41-50.

9. Omura M, Sasano H, Fujiwara T, Yamaguchi K, Nishikawa T. Unique cases of unilateral hyperaldosteronemia due to multiple adrenocortical micronodules, which can only be detected by selective adrenal venous sampling. Metabolism: Clin Exp 2002;51(3):350-355.

10. Goh BK, Tan YH, Chang KT, Eng PH, Yip SK, Cheng CW. Primary hyperaldosteronism secondary to unilateral adrenal hyperplasia: an unusual cause of surgically correctable hypertension. A review of 30 cases. World J Surg. 2007;31(1):72-79.

11. Funder JW, Carey RM, Mantero F, Murad MH, Reincke M, Shibata H, Stowasser M, Young WF Jr. The management of primary aldosteronism: case detection, diagnosis, and treatment: an endocrine society clinical practice guideline. J Clin Endocrinol Metab 2016;101(5):1889-1916.

12. Nishikawa T, Omura M, Satoh F, Shibata H, Takahashi K, Tamura N, Tanabe A. Guidelines for the diagnosis and treatment of primary aldosteronism--the Japan Endocrine Society 2009. Endocr J 2011;58(9):711-721.

13. Rossi GP, Barisa M, Allolio B, Auchus RJ, Amar L, Cohen D, Degenhart C, Deinum J, Fischer E, Gordon R, Kickuth R, Kline G, Lacroix A, Magill S, Miotto D, Naruse M, Nishikawa T, Omura M, Pimenta E, Plouin PF, Quinkler M, Reincke M, Rossi E, Rump LC, Satoh F, Schultze Kool L, Seccia TM, Stowasser M, Tanabe A, Trerotola S, Vonend O, Widimsky J, Jr, Wu KD, Wu VC, Pessina AC. The Adrenal Vein Sampling International Study (AVIS) for identifying the major subtypes of primary aldosteronism. J Clin Endocrinol Metab 2012;97(5):1606-1614.

14. Rossi GP, Auchus RJ, Brown M, Lenders JW, Naruse M, Plouin PF, Satoh F, Young WF Jr. An expert consensus statement on use of adrenal vein sampling for the subtyping of primary aldosteronism. Hypertension 2014;63(1):151-160.

15. Monticone S, Viola A, Rossato D, Veglio F, Reincke M, GomezSanchez C, Mulatero P. Adrenal vein sampling in primary aldosteronism: towards a standardised protocol. Lancet Diabetes Endocrinol. 2015;3(4):296-303.

16. Rossi GP, Rossitto G, Amar L, Azizi M, Riester A, Reincke M, Degenhart C, Widimsky J Jr, Naruse M, Deinum J, Schultzekool LJ, Kocjan T, Negro A, Rossi E, Kline G, Tanabe A, Satoh F, Rump LC, Vonend O, Willenberg HS, Fuller P, Yang J, Nian Chee NY, Magill SB, Shafigullina Z, Quinkler M, Oliveras A, Wu KD, Wu VC, Somloova Z, Barbiero G, Battistel M, Chang C-C, Vanderriele PE, Pessina AC. The clinical outcomes of 1625 subtyped patients with primary aldosteronism and with/without successful adrenal vein sampling. Hypertension 2019;74(4):800-808.

17. Subtyping of Primary Aldosteronism in the AVIS-2 Study: Assessment of Selectivity and Lateralization - SUPPLEMENTAL MATERIAL. 2019. http://researchdata.cab.unipd.it/id/eprint/345. Accessed July 27, 2019.

18. Rossi GP, Pitter G, Bernante P, Motta R, Feltrin G, Miotto D. Adrenal vein sampling for primary aldosteronism: the assessment of selectivity and lateralization of aldosterone excess baseline and after adrenocorticotropic hormone (ACTH) stimulation. $J$ Hypertens 2008;26(5):989-997.

19. Rossitto G, Miotto D, Battistel M, Barbiero G, Maiolino G, Bisogni V, Sanga V, Rossi GP. Metoclopramide unmasks potentially misleading contralateral suppression in patients undergoing adrenal vein sampling for primary aldosteronism. J Hypertens 2016;34(11):2258-2265.

20. Espiner EA, Ross DG, Yandle TG, Richards AM, Hunt PJ. Predicting surgically remedial primary aldosteronism: role of adrenal scanning, posture testing, and adrenal vein sampling. J Clin Endocrinol Metab 2003;88(8):3637-3644.

21. Rossitto G, Maiolino G, Lenzini L, Bisogni V, Seccia TM, Cesari M, Iacobone M, Rossi GP. Subtyping of primary aldosteronism with adrenal vein sampling: Hormone- and 
side-specific effects of cosyntropin and metoclopramide. Surgery 2018;163(4):789-795.

22. Williams TA, Lenders JWM, Mulatero P, Burrello J, Rottenkolber M, Adolf C, Satoh F, Amar L, Quinkler M, Deinum J, Beuschlein F, Kitamoto KK, Pham U, Morimoto R, Umakoshi H, Prejbisz A, Kocjan T, Naruse M, Stowasser M, Nishikawa T, Young WF Jr, GomezSanchez CE, Funder JW, Reincke M. Outcomes after adrenalectomy for unilateral primary aldosteronism: an international consensus on outcome measures and analysis of remission rates in an international cohort. Lancet Diabetes Endocrinol. 2017;5(9):689-699.

23. Funder JW, Carey RM, Fardella C, Gomez-Sanchez CE, Mantero F, Stowasser M, Young WF Jr, Montori VM. Case detection, diagnosis, and treatment of patients with primary aldosteronism: an endocrine society clinical practice guideline. J Clin Endocrinol Metab 2008;93(9):3266-3281.

24. Seccia TM, Miotto D, De Toni R, Gallina V, Vincenzi M, Pessina AC, Rossi GP. Subtyping of primary aldosteronism by adrenal vein sampling: effect of acute $\mathrm{D}(2)$ receptor dopaminergic blockade on adrenal vein cortisol and chromogranin A levels. Eur $J$ Endocrinol 2011;165(1):85-90.

25. Seccia TM, Miotto D, De Toni R, Pitter G, Mantero F, Pessina AC, Rossi GP. Adrenocorticotropic hormone stimulation during adrenal vein sampling for identifying surgically curable subtypes of primary aldosteronism: comparison of 3 different protocols. Hypertension 2009;53(5):761-766.

26. Miotto D, De Toni R, Pitter G, Seccia TM, Motta R, Vincenzi M, Feltrin G, Rossi GP. Impact of accessory hepatic veins on adrenal vein sampling for identification of surgically curable primary aldosteronism. Hypertension 2009;54(4):885-889.

27. Omura K, Ota H, Takahashi Y, Matsuura T, Seiji K, Arai Y, Morimoto R, Satoh F, Takase K. Anatomical variations of the right adrenal vein: concordance between multidetector computed tomography and catheter venography. Hypertension 2017;69(3):428-434.

28. El Ghorayeb N, Mazzuco TL, Bourdeau I, Mailhot JP, Zhu PS, Therasse E, Lacroix A. Basal and post-ACTH aldosterone and its ratios are useful during adrenal vein sampling in primary aldosteronism. J Clin Endocrinol Metab 2016;101(4):1826-1835.

29. Dekkers T, Prejbisz A, Kool LJS, Groenewoud H, Velema M, Spiering W, Kolodziejczyk-Kruk S, Arntz M, Kadziela J, Langenhuijsen JF, Kerstens MN, van den Meiracker AH, van den Born BJ, Sweep F, Hermus A, Januszewicz A, LigthartNaber AF, Makai P, van der Wilt GJ, Lenders JWM, Deinum J. Adrenal vein sampling versus CT scan to determine treatment in primary aldosteronism: an outcome-based randomised diagnostic trial. Lancet Diabetes Endocrinol. 2016;4(9):739-746.

30. Dekkers T, Deinum J, Schultzekool LJ, Blondin D, Vonend O, Hermus AR, Peitzsch M, Rump LC, Antoch G, Sweep FC, Bornstein SR, Lenders JW, Willenberg HS, Eisenhofer G. Plasma metanephrine for assessing the selectivity of adrenal venous sampling. Hypertension 2013;62(6):1152-1157.

31. Eisenhofer G, Dekkers T, Peitzsch M, Dietz AS, Bidlingmaier M, Treitl M, Williams TA, Bornstein SR, Haase M, Rump LC, Willenberg HS, Beuschlein F, Deinum J, Lenders JW, Reincke M. Mass spectrometry-based adrenal and peripheral venous steroid profiling for subtyping primary aldosteronism. Clinical Chemistry 2016;62(3):514-524.

32. Ceolotto G, Antonelli G, Maiolino G, Cesari M, Rossitto G, Bisogni V, Plebani M, Rossi GP. Androstenedione and 17-alpha-hydroxyprogesterone are better indicators of adrenal vein sampling selectivity than cortisol. Hypertension 2017;70(2):342-346. 